\title{
DNA Sequencing Modified Method through Effective Regulation of Its Translocation Speed in Aqueous Solution
}

\author{
Lusine Gasparyan1,2, Ilya Mazo³, Ferdinand Gasparyan1, Vahan Simonyan² \\ ${ }^{1}$ Yerevan State University, Yerevan, Armenia \\ ${ }^{2}$ DNA-HIVE LLC, 15313 Diamond Cove Terrace, Rockville, MD, USA \\ ${ }^{3}$ Argentis LLC, Gaithersburg, MD, USA \\ Email: fgaspar@ysu.am
}

How to cite this paper: Gasparyan, L., Mazo, I., Gasparyan, F. and Simonyan, V. (2020) DNA Sequencing Modified Method through Effective Regulation of Its Translocation Speed in Aqueous Solution. Open Journal of Biophysics, 10, 96-112.

https://doi.org/10.4236/ojbiphy.2020.102009

Received: March 18, 2020

Accepted: April 23, 2020

Published: April 26, 2020

Copyright $\odot 2020$ by author(s) and Scientific Research Publishing Inc. This work is licensed under the Creative Commons Attribution International License (CC BY 4.0).

http://creativecommons.org/licenses/by/4.0/

\begin{abstract}
Solid-state nanopore DNA sequencing modified method is developed. Method is based on the tunnel current investigation through the nanogap made on lateral gold electrodes in the form of nanowires or nanoribbons. The movement of DNA in aqueous solution is regulated by the potential applied to reference electrode. The potential applied to the lateral metal electrodes helps to the creation of the molecular junctions. They consist of the nucleosides passing through the pores. Taking into account that DNA moves under gravity, electrophoretic and drag forces, the analytic expression for the DNA translocation speed is calculated and analyzed. The conditions for decreasing the DNA translocation speed or increasing the nucleosides reading time are received. It is shown that one can control value of the DNA molecules bases reading time and the frequency of the bases passes by the choice of magnitude of the potential applied to reference electrode. Our results, therefore potentially suggest a realistic, inherently design-specific, high-throughput nanopore DNA sequencing device/cell as a de-novo alternative to the existing methods.
\end{abstract}

\section{Keywords}

DNA Sequencing, DNA Speed, Forces, Reading Time

\section{Introduction}

The process of DNA sequencing is a precise determination of the amount and distribution of the nucleotides in DNA molecules. Quick improvements in the cost and speed of DNA sequencing are having a strong influence on comprehen- 
sive genome research. Methods of DNA sequencing and their application are detailed analyzed in [1]. In the past decade, the single-nanometer-scale pores demonstrated great capability for the detection, identification, and characterization of DNA [2] [3] and RNA [4] [5]. In recent years, rapid advances have been made and many construction's architectures have been put forward for novel approaches to bio-molecular sensing using nanoelectronics, including the advent of tunnel junctions as a sensing platform. Within the past decade, nanogap electrodes have attracted a lot of attention because of their potential applications in the progressive miniaturization of electronics and as nanoscale tool for analysis of single molecular properties. DNA bases can be identified statistically in nanopore translocation events. Nanogap electrodes can be simply defined as a pair of electrodes with a gap that can be measured in nanometers. The transport dynamics of the charged molecules in the nanosize constructions located in aqueous solution environment is the result of multiple factors including, electrostatic and hydrodynamic interactions, drift and diffusion.

One major challenge of nanopore-based DNA sequencing technology is to find an efficient way to reduce DNA translocation speed. It is necessary that each nucleotide can reside long enough in the measuring pore for creation molecular junctions. Magnitudes of those current will help identification of nucleotides [6]. In order to take control of the DNA molecule translation process, various theories and approaches have been put forward in recent decades.

Authors of Ref. [7] considered that the reasonable value of the DNA translocation speed is near to $0.01-1 \mathrm{~ms}$ per base, which is equivalent to (10 - 1000) bases/s. Peng and Ling reversed the DNA translocation and achieved an average speed of 0.0096 bases/ $\mu \mathrm{s}=9600$ bases/s [8]. As the translocation times depend strongly on the nucleotide type [9], imply that polymer-pore interactions, rather than the more generic hydrodynamic drag, play an important role in determining the translocation dynamics. The interaction of the polymer with pores is described in detail in [9]. In [10], the authors propose a feedback device architecture for regulating DNA translocation by modulating the effective surface charge density of a nanopore wall. It was shown that the rate of DNA movement can be reduced at a rate of about $55 \mathrm{~mm} / \mathrm{s}$ per $1 \mathrm{mV} / \mathrm{nm}$. The review [11] focuses on a single aspect in the transport dynamics of a polymer drawn inside a nanoscopic channel. Primarily, the dynamics of polynucleotides is discussed. Some of the concepts that are discussed in [11] apply to uncharged polymers.

Thus, a key challenge to DNA sequencing with nanopores is to find methods to slow down and control DNA translocation. DNA translocation speeds can be reduced somewhat by decreasing temperature [12] [13], or increasing solvent viscosity [7] [14], but these methods do not reduce the variations in the translocation dynamics because of DNA-pore interactions [15] [16] [17] [18]. Problems of controlling DNA motion and translocation in a nanopore analyzed also in [19]. The detailed analyses of the literature data, some critical considerations and the potential ways of optimization of DNA nanopore sequencing were presented 
in [20]. Some characteristics of ISFET and EIS based DNA sensors are analyzed detailed by us in [21] [22].

In this paper a modified architecture/design for measuring cell of DNA sequencing using tunneling current is offered in order to control and optimize the translocation speed of the DNA molecules. We study the features of the dynamics of the movement of DNA molecule in an aqueous solution under gravity, electrophoretic and drag forces in order to reduce the rate of movement of the DNA and increase DNA reading time.

\section{DNA Dynamics and DNA Speed in Aqueous Solution}

In Figure 1 the scheme of architecture/design for investigation of DNA nucleosides sequencing by the solid state nanopore modified method is presented. The electrolytic cell filled by aqueous solution provides with nanopore, reference and back electrodes (RE and $\mathrm{BE}$ ). The electrical potential applied on the RE/BE promotes directional movement of the charged DNA molecules under the action of an electric field. The potential applied to the lateral metal electrodes M1 and M2 facilitates the creation of molecular junctions with nucleosides. A nanopore consisting of gold electrodes is covered by insulator layer. Contacts to nanopore can be made in the form of nanowires or nano-ribbons (Figure 1(c)). Contacts surface area coated by the insulator layer for its protection and for not letting the negatively charged molecules stick the surface of the electrodes. Capture of DNA molecules on the gold electrode surface will create "false" currents and distort the useful signal. If necessary, many nanopores can be mounted in a cell and a multi-nanopore chamber can be created and at the same time many DNA molecules can be studied (Figure 1(b)). In Figure 1(d) example of molecular junction M1-DNA GC base pair-M2 is presented.

At the bare pore under applied voltage $V$ between metallic electrodes M1 and M2 flow only low ionic current of electrolyte $I_{i \perp}$ in order to pA [6] [23] [24]. Ionic current through the channel sharply decreases (is blockade) while the DNA molecule move under the gravity $\boldsymbol{P}$, electrophoretic force $\boldsymbol{F}_{\boldsymbol{e}}$ (migration under applied potential on the reference electrode), drag force $\boldsymbol{F}_{\boldsymbol{D}}$ and diffusion (connected with ions concentration gradient in solution) become to the pore and blockade the ionic current. When some of nucleotides of DNA [adenine (A), guanine (G), thymine (T), cytosine (C), nucleotide pairs GC, AT, etc.] captured between gold electrodes, they create the electronic bridge and consequently molecular junctions (at the $V \neq 0$, see [6]). So, DNA bases can create individual electronic bridges between gold electrodes and across a pore (through the nucleotide junction) that will flow only tunnel current. Metal electrodes can be made in the form of nanowires/nanoribbons to create, if possible, a large area of coverage of the coming close DNA molecules with several orientations (Figure 1(c)). Such a design of the electrodes can be realized using mechanically controllable break-junction (MCBJ) [25] [26] [27], scanning tunnel microscope (STM) [28] or atomic force microscope (AFM) [29] techniques. 


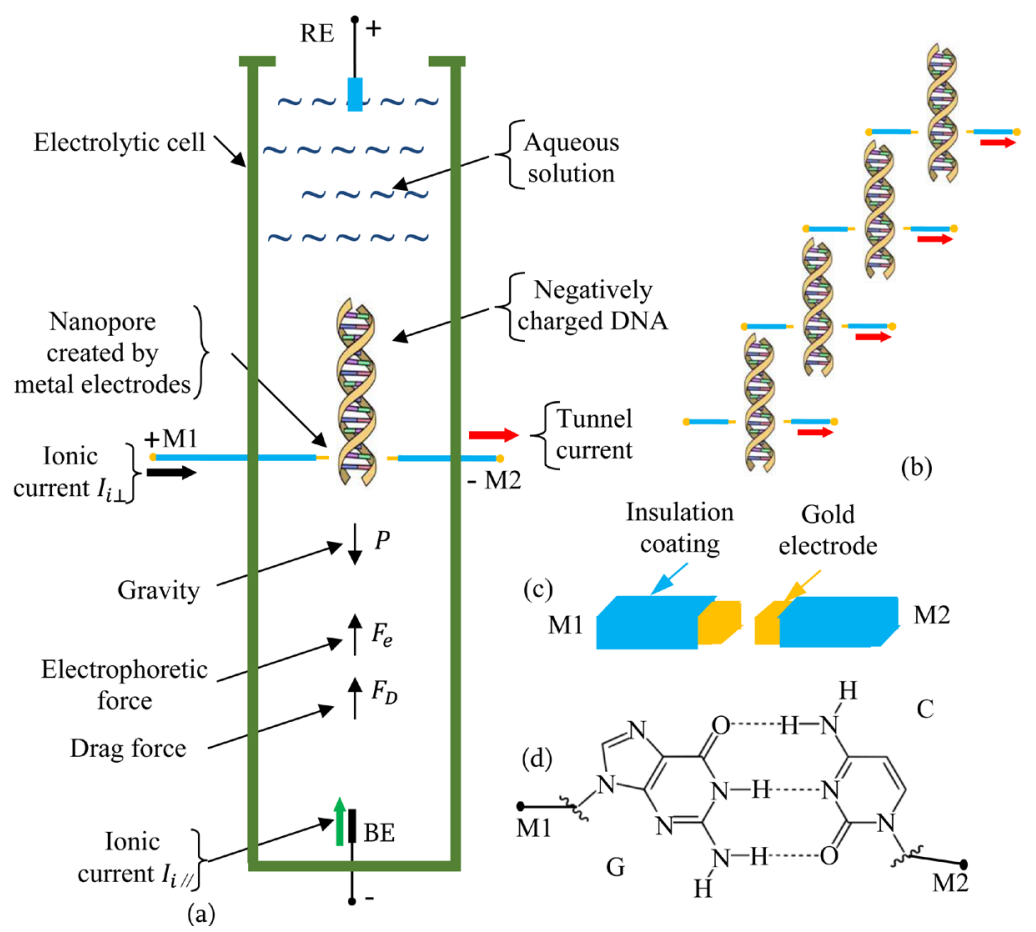

Figure 1. (a) The measurement cell of investigation of DNA nucleosides by the solid state nanopore modified method. (b) Multi-nanopore (4 nanopore) design. (c) Nanogape with nanowire or nanoribbon gold electrodes. (d) Example of molecular junction M1-DNA GC base pair-M2. The potential applied to the electrodes $[\mathrm{Au}(111)]$ facilitates the creation of a molecular junction with nucleosides. The $\mathrm{Au}(111)$ substrate was chosen as a noble inert substrate to minimize molecule-substrate interactions. Vertical ionic current $I_{i \|}$ depend with potential $V_{0}$, applied to RE. Lateral ionic current $I_{i \perp}$ conditioned by potential applied to metallic electrodes. RE means reference electrode, BE means back electrode. It is showing also the gravity $(\boldsymbol{P})$, electrophoretic $\left(\boldsymbol{F}_{e}\right)$ and $\operatorname{drag}\left(\boldsymbol{F}_{\boldsymbol{D}}\right)$ forces, influences on molecules and controlled their movement.

Such a design of electrodes will contribute to almost $\sim 100$ percent creation of molecular junctions at any orientation of the DNA. The gap between electrodes must be so narrow that only one DNA molecule passes through it.

For effective reading of nucleotides, it is necessary that they move vertically. The vertically directional movement of the molecule will be determined by the resulting force $\boldsymbol{F}$

$$
\boldsymbol{F}=\boldsymbol{P}+\boldsymbol{F}_{\boldsymbol{e}}+\boldsymbol{F}_{\boldsymbol{D}}
$$

Let's assume that distribution of the DNA molecules in aqueous solution is homogeneous and DNA concentration gradient in the solution is absence or very small. Then ignoring the role of diffusion and considering that

$$
|\boldsymbol{P}|=m_{0} g,\left|\boldsymbol{F}_{\boldsymbol{e}}\right|=q \mathcal{E}_{0}=\frac{q V_{0}}{l_{0}}, \quad\left|\boldsymbol{F}_{\boldsymbol{D}}\right|=\frac{1}{2} \rho v^{2} C_{D} A,
$$

for the magnitude of the resulting force we can write:

$$
F=m_{0} g+\left|q \mathcal{E}_{0}\right|-\frac{1}{2} \rho v^{2} C_{D} A .
$$


Here $m_{0}$ is the mass of DNA molecule, $V_{0}$ is the applied voltage on the reference electrode, $l_{0}$ is the distance between RE and nanogap, $\mathcal{E}_{0}=V_{0} / l_{0}$ is the electric field acting on DNA, $g=9.80665 \mathrm{~m} / \mathrm{s}^{2}, q$ is the negative charge of DNA, $v$ is the speed of the molecule relative to the aqueous solution, $A$ is the molecule cross sectional area, $\rho$ is the density of the solution ${ }^{1}$, and $C_{D}$ is the drag coefficient-a dimensionless number ${ }^{2}$.

Let's discuss the magnitudes of $|\boldsymbol{P}|,\left|\boldsymbol{F}_{\boldsymbol{e}}\right|$ and $\left|\boldsymbol{F}_{\boldsymbol{D}}\right|$.

For definiteness assume that DNA consists only from $N_{n}$ blocks of A, G, C and T nucleotides. Then for $m_{0}$ we have:

$$
\begin{aligned}
m_{0}= & N_{n}\left(m_{A}+m_{G}+m_{T}+m_{C}\right)=\frac{N_{n}}{N_{A}}\left(M_{A}+M_{G}+M_{T}+M_{C}\right) \\
= & \frac{N_{n}}{N_{A}} \times\left[M_{A}\left(\mathrm{C}_{5} \mathrm{H}_{5} \mathrm{~N}_{5}\right)+M_{G}\left(\mathrm{CH}_{5} \mathrm{~N}_{5} \mathrm{O}\right)+M_{T}\left(\mathrm{C}_{5} \mathrm{H}_{6} \mathrm{~N}_{2} \mathrm{O}_{2}\right)+M_{C}\left(\mathrm{C}_{4} \mathrm{H}_{5} \mathrm{~N}_{3} \mathrm{O}\right)\right] \\
= & \frac{N_{n}}{N_{A}} \times[5 \times(12+1+14)+(12+5 \times 1+5 \times 14+16) \\
& +(5 \times 12+6 \times 1+2 \times 14+2 \times 16)+(4 \times 12+5 \times 1+3 \times 14+16)] \\
\approx & 8 \times 10^{-22} N_{n} \mathrm{~g}=m_{n} N_{n} \mathrm{~kg} \\
m_{n}= & 8 \times 10^{-25} \mathrm{~kg} .
\end{aligned}
$$

Here $N_{A}$ is the Avogadro number, $m_{n}$ is the mass of group from four nucleotides, $M_{A}, M_{G}, M_{T}$ and $M_{C}$ are the molecular masses of adenine $\left(\mathrm{C}_{5} \mathrm{H}_{5} \mathrm{~N}_{5}\right)$, guanine $\left(\mathrm{CH}_{5} \mathrm{~N}_{5} \mathrm{O}\right)$, thymine $\left(\mathrm{C}_{5} \mathrm{H}_{6} \mathrm{~N}_{2} \mathrm{O}_{2}\right)$ and cytosine $\left(\mathrm{C}_{4} \mathrm{H}_{5} \mathrm{~N}_{3} \mathrm{O}\right)$, correspondingly. For example, at the $N_{n}=1000$, we have $m_{0} \approx 8 \times 10^{-22} \mathrm{~kg}$ and

$$
|\boldsymbol{P}|=7.84 \times 10^{-21} \mathrm{~N} .
$$

The value of $F_{e}$ depends on $\mathcal{E}_{0}$ and for example at the single charged DNA $\left(q \equiv e=1.6 \times 10^{-19} \mathrm{C}\right), \quad V_{0}=2 \mathrm{~V}$ and at the $l_{0}=1 \mathrm{~cm}$ for the $\left|\boldsymbol{F}_{\boldsymbol{e}}\right|$ we have:

$$
\left|\boldsymbol{F}_{e}\right|=\frac{q V_{0}}{l_{0}}=3.2 \times 10^{-21} \mathrm{~N} \text {. }
$$

Lets' consider the value $F_{D}$. Taking $\rho \approx 1 \mathrm{~g} / \mathrm{cm}^{3}=10^{3} \mathrm{~kg} / \mathrm{m}^{3}$ (as a water, $\mathrm{pH}=7), \quad C_{D} \approx 0.42[9], \quad A=\frac{\pi d^{2}}{4} \approx 3.8 \times 10^{-18} \mathrm{~m}^{2} \quad(d \approx 2.2 \mathrm{~nm}$ is the DNA diameter [31]) we receive

${ }^{1}$ Density of the fluid determined by the expression $\rho=3.446 \delta \times 10^{-3}+1.0048 \mathrm{~g} / \mathrm{ml} \quad$ [30], where $\delta$ is the percent composition. Density of the fluid changed very little, e.g. at the $0<\delta \leq 20$, $1<\rho \leq 1.1 \mathrm{~g} / \mathrm{ml}$.

${ }^{2}$ The drag coefficient $C_{D}$ depends on the shape of the object and on the Reynolds number $R_{e}=v D / v_{0}$. Here $D$ is some characteristic diameter or linear dimension and $v_{0}$ is the kinematic viscosity of the fluid. At low $R_{e} C_{D}$ is asymptotically proportional to $R_{e}^{-1}$, which means that the drag is linearly proportional to the speed. At high $R_{e} C_{D}$ is more or less constant and drag will vary as the square of the speed. In the case of high velocity, in general, $C_{D}$ a function of the orientation of the flow with respect to the molecule (apart from symmetrical objects like a sphere). Assuming that surface area of the DNA molecule end is the half-sphere in further calculations we can take $C_{D} \approx 0.42[9]$. 


$$
\left|\boldsymbol{F}_{\boldsymbol{D}}\right| \approx 7.98 \times 10^{-16} v^{2} \mathrm{~N}
$$

Where molecule speed $v$ given in $\mathrm{m} / \mathrm{s}$.

Now let's determine functional dependency of molecule translocation speed $v$ vs. other parameters, especially vs. $V_{0}$ and $l_{0}$. Molecule move under resulting force (3). Kinetic energy of the molecule is equal to work of resulting force. Taking the aqueous solution surface as the origin of coordinate and assume that initial coordinate of molecule is the $l_{i}$ (Figure 1) and DNA initial speed is $v_{i}$ we can write

$$
\int_{l_{i}}^{l_{0}} F \mathrm{~d} z=\frac{m_{0}\left(v-v_{i}\right)^{2}}{2}
$$

Then on the distance $\left(l_{0}-l_{i}\right)$ molecule will accumulate the kinetic energy equal to work of resulting force and

$$
F\left(l_{0}-l_{i}\right)=\frac{m_{0}\left(v-v_{i}\right)^{2}}{2},
$$

or

$$
m_{0} g+\left|q \mathcal{E}_{0}\right|-\frac{1}{2} \rho v^{2} C_{D} A=\frac{m_{0}\left(v-v_{i}\right)^{2}}{2\left(l_{0}-l_{i}\right)},
$$

and

$$
v-v_{i}=\left[\frac{2\left(m_{0} g \mp q \mathcal{E}_{0}\right)}{m_{0} /\left(l_{0}-l_{i}\right)+\rho C_{D} A}\right]^{1 / 2} .
$$

One of significant problems of DNA sequencing is to decrease translocation speed. Assume that molecule starts moving from upper side of chamber $\left(l_{i}=0\right)$ with zero starting speed $\left(v_{i}=0\right)$. For regulating the DNA speed as variable parameters, we can choose $l_{0}$ and $\mathcal{E}_{0}$. It is clear also that $v>0$ and for minimizing $v$ we must put "-" sign in (8) before $q \mathcal{E}_{0}$ and consider the condition

$$
\left|m_{0} g\right|>\left|q \mathcal{E}_{0}\right| \quad \text { or } \quad \mathcal{E}_{0}=\frac{V_{0}}{l_{0}}<\frac{m_{0} g}{q} .
$$

Then expression (8) we can rewrite as

$$
v=\left[\frac{2\left(m_{0} g-q \mathcal{E}_{0}\right)}{m_{0} / l_{0}+\rho C_{D} A}\right]^{1 / 2} .
$$

At the $m_{0} \approx 8 \times 10^{-22} \mathrm{~kg}\left(N_{n}=10^{3}\right), \quad q \equiv e=1.6 \times 10^{-19} \mathrm{C}$ and $g \approx 10 \mathrm{~m} / \mathrm{s}^{2}$ the condition (9) equivalent to:

$$
\mathcal{E}_{0}<5 \times 10^{-2} \mathrm{~V} / \mathrm{m} \text {. }
$$

Measurements of blockade current of ssDNA in [32] imply that, while polymers longer than the pore they are translocated at a constant speed, the velocity of shorter polymers increases with decreasing length. Note that unlike our case in [32] polarity of the potential applied to gate electrode and electric force directed to opposite (Figure 1(a) in [32]), and velocity quadratically depends on applied field. 
As we can see the magnitude of DNA translocation speed strongly depend on $\mathcal{E}_{0}$ and the DNA size, particularly on $N_{n}$. Using the above parameters, we obtain

$$
\frac{m_{n} N_{n}}{\rho C_{D} A l_{0}} \approx 5 \times 10^{-8} N_{n} .
$$

For the "optimal" case $N_{n}<2 \times 10^{8}$ from (10) we have

$$
v \propto \sqrt{N_{n}} .
$$

The schematic behaviors of the molecule speed vs. $\mathcal{E}_{0}$ and $N_{n}$ are shown in Figure 2. DNA translocation speed dramatically decreases with the electric field. About such behavior of $v\left(\mathcal{E}_{0}\right)$ is noted also in [10] [11]. Note that the calculated results of [11] predict that for long polymers the translocation mean velocity, defined as the ratio of the polymer contour length and the average first passage time, approaches a constant value that does not depend on $N_{n}$. This type of dependence is consistent with our dependencies at the higher values of $N_{n}$ (saturation of $v\left(N_{n}\right)$ ) (Figure 2(b)).

For receiving low value of speed, we need to minimalized term $\left(m_{0} g-q \mathcal{E}_{0}\right)$ by the varying $\mathcal{E}_{0}$ and $N_{n}$. Problem is to find optimal values of $\mathcal{E}_{0}$.

Let's introduce dimensionless electric field strength $\mathcal{E}$ and dimensionless speed $v_{0}$ as follows:

$$
\mathcal{E} \equiv \frac{q \mathcal{E}_{0}}{m_{0} g}, \quad v_{0} \equiv \frac{v}{\sqrt{2 g l_{0}}} \sqrt{1+\frac{\rho C_{D} A l_{0}}{m_{0}}} .
$$

Now expression (10) we can rewrite as follows:

$$
v_{0}=(1-\mathcal{E})^{1 / 2}, \quad \text { or } \quad \lg v_{0}=\frac{1}{2} \lg (1-\mathcal{E})
$$

For receive low speed it is necessary that $(1-\mathcal{E})$ will be minimal, or with values of $\mathcal{E}$ very close to unity.

Numerical calculations of the DNA translocation speed vs. $(1-\mathcal{E})$ are carried out. Results of numerical analyzes for DNA three sizes $\left(N_{n}=10^{3}, N_{n}=40\right.$ and $N_{n}=10$ ) presented in Table 1 . Low magnitude of $v$ is obtained with

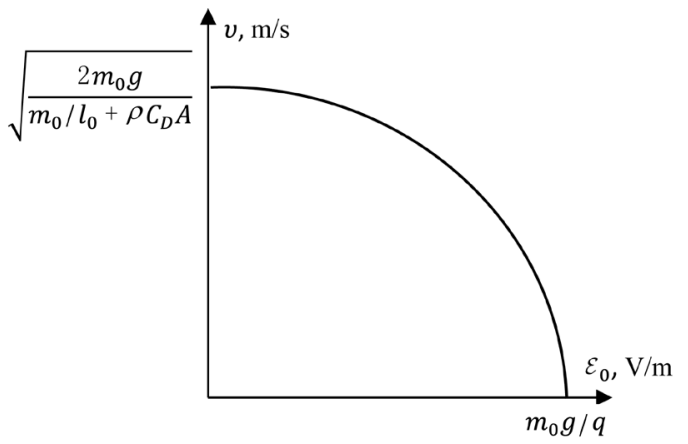

(a)

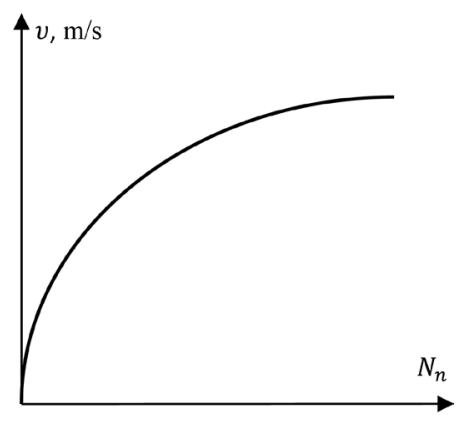

(b)

Figure 2. Schematic dependency of the molecule speed vs. $\mathcal{E}_{0}$ (a) and $N_{n}$ (b). 
Table 1. Numerical data of the DNA translocation speed for cases $N_{n}=1000,40$ and 10 .

\begin{tabular}{|c|c|c|c|c|c|c|}
\hline \multicolumn{7}{|c|}{$N_{n}=1000$} \\
\hline $1-\mathcal{E}$ & $10^{-5}$ & $10^{-6}$ & $10^{-7}$ & $10^{-8}$ & $10^{-9}$ & $10^{-10}$ \\
\hline $\lg (1-\mathcal{E})$ & -5 & -6 & -7 & -8 & -9 & -10 \\
\hline $\mathcal{E}_{0}, \mathrm{mV} / \mathrm{cm}$ & 0.0499995 & 0.04999995 & 0.049999995 & 0.04999999995 & 0.04999999995 & 0.0499999999995 \\
\hline $\lg v_{0}$ & $-5 / 2$ & -3 & $-7 / 2$ & -4 & $-9 / 2$ & -5 \\
\hline$v_{0}$ & $10^{-5 / 2}$ & $10^{-3}$ & $10^{-7 / 2}$ & $10^{-4}$ & $10^{-9 / 2}$ & $10^{-5}$ \\
\hline$v, \mathrm{~nm} / \mathrm{s}$ & $3.166 \times 10^{7 / 2}=10^{4}$ & $3.166 \times 10^{3}$ & $3.166 \times 10^{5 / 2}=10^{3}$ & $3.166 \times 10^{2}$ & $\left.3.166 \times 10^{3 / 2}=10^{2}\right)$ & 31.66 \\
\hline \multicolumn{7}{|c|}{$N_{n}=40$} \\
\hline $1-\mathcal{E}$ & $10^{-5}$ & $10^{-6}$ & $10^{-7}$ & $10^{-8}$ & $10^{-9}$ & $10^{-10}$ \\
\hline $\lg (1-\mathcal{E})$ & -5 & -6 & -7 & -8 & -9 & -10 \\
\hline $\mathcal{E}_{0}, \mathrm{~V} / \mathrm{cm}$ & 0.0499995 & 0.04999995 & 0.049999995 & 0.0499999995 & 0.04999999995 & 0.0499999999995 \\
\hline $\lg v_{0}$ & $-5 / 2$ & -3 & $-7 / 2$ & -4 & $-9 / 2$ & -5 \\
\hline$v_{0}$ & $10^{-5 / 2}$ & $10^{-3}$ & $10^{-7 / 2}$ & $10^{-4}$ & $10^{-9 / 2}$ & $10^{-5}$ \\
\hline$v, \mathrm{~nm} / \mathrm{s}$ & $\left.6.33 \times 10^{5 / 2}=2000\right)$ & $6.33 \times 10^{2}=633$ & $6.33 \times 10^{3 / 2}=200$ & 63.3 & 20 & 6.33 \\
\hline \multicolumn{7}{|c|}{$N_{n}=10$} \\
\hline $1-\mathcal{E}$ & $10^{-5}$ & $10^{-6}$ & $10^{-7}$ & $10^{-8}$ & $10^{-9}$ & $10^{-10}$ \\
\hline $\lg (1-\mathcal{E})$ & -5 & -6 & -7 & -8 & -9 & -10 \\
\hline $\mathcal{E}_{0}, \mathrm{~V} / \mathrm{cm}$ & 0.0499995 & 0.04999995 & 0.049999995 & 0.0499999995 & 0.04999999995 & 0.049999999995 \\
\hline $\lg v_{0}$ & $-5 / 2$ & -3 & $-7 / 2$ & -4 & $-9 / 2$ & -5 \\
\hline$v_{0}$ & $10^{-5 / 2}$ & $10^{-3}$ & $10^{-7 / 2}$ & $10^{-4}$ & $10^{-9 / 2}$ & $10^{-5}$ \\
\hline$v, \mathrm{~nm} / \mathrm{s}$ & $3.166 \times 10^{5 / 2}=10^{3}$ & 317 & $3.166 \times 10^{3 / 2}=100$ & 32 & $\left.3.166 \times 10^{1 / 2}=10\right)$ & 3 \\
\hline
\end{tabular}

low values of $\mathcal{E}_{0}$ and for short DNA (low values of $N_{n}$ ). For comparison in Table 2 is presented some literature data of DNA translocation speed.

The comparison of the results of Table 1 and Table 2 shows the advantage of the proposed method of reducing the translocation speed. Speed control is achieved through the accurate compensation of gravity and drag forces. This can be achieved by controlling the potential of the reference electrode. By adjusting the size of DNA molecule and increasing the accuracy of changing of the reference electrode potential, the translocation speed can be significantly reduced (see cases $N_{n}=40$ or 10 ).

In Figure 3 the logarithmic dependency of dimensionless DNA translocation speed vs. dimensionless field $\mathcal{E}$ is presented for the case $N_{n}=40$ according to data of Table 1. This graph built for the case of the optimal values of $V_{0}=0.001 \mathrm{~V}$ and $l_{0}=0.02 \mathrm{~m}$. In this linear dependency it also shows corresponding values of DNA molecule translocation speed in $\mathrm{nm} / \mathrm{s}$ units for several values of $\lg (1-\mathcal{E})$.

The main difficulty of such way to decrease translocation speed conditioned 
Table 2. Literature data of DNA translocation speed.

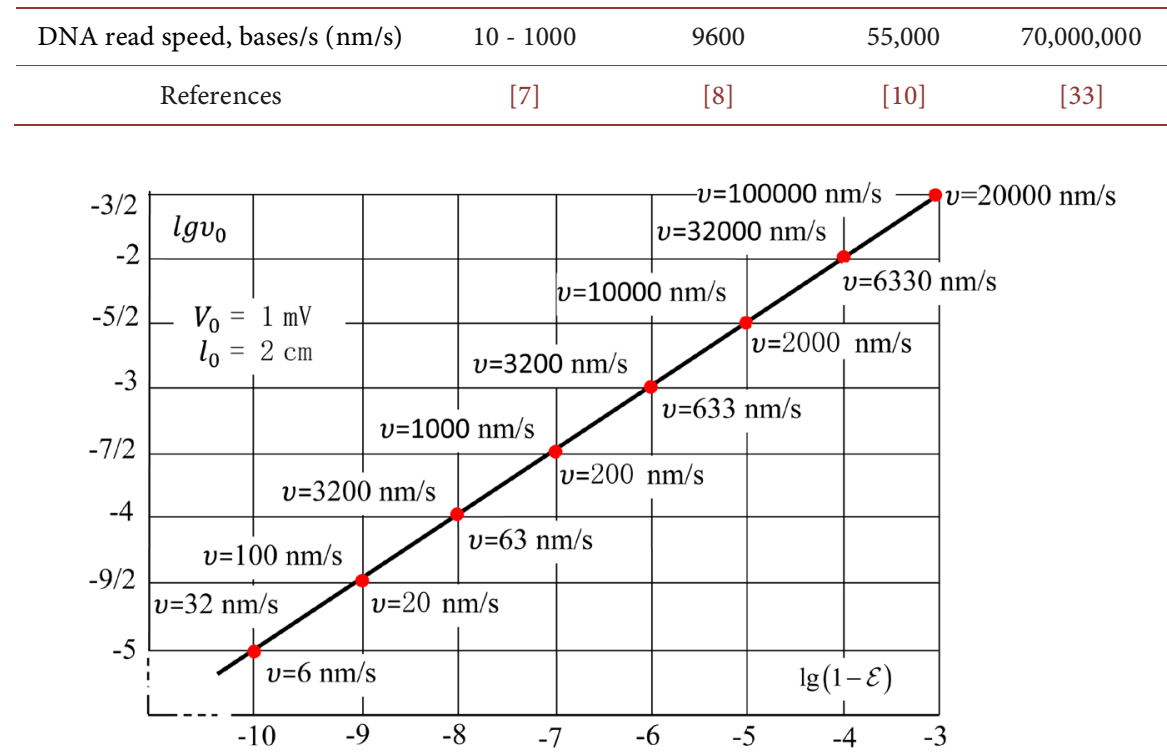

Figure 3. Logarithmic dependency of DNA translocation dimensionless speed vs. dimensionless electric field. It is shown values of molecules speed in $\mathrm{nm} / \mathrm{s}$ for the case $N_{n}=40$ (the data on the right site) and $N_{n}=1000$ (the data on the left site).

by the very slightly changing of electric field $\mathcal{E}_{0}$. The translocation velocity varies also depend on parameters such as the electrical potential, the type of nanopore, and whether the DNA is single-stranded or double-stranded (see also [34]).

\section{Elevator Method}

An alternative method for reducing DNA translocation time can be the so-called "elevator method". By the elevator method, we mean the situation when the nanopore construction (metallic electrodes) moves parallel to $Z$ axis along with the DNA (Figure 1). Moreover, the speed of movement of a nanopore must be approximately equal to or slightly weaker than the speed of movement of DNA. The "elevator method" can be easily implemented in Space (in the Shuttle) without gravity. At the same time, the depth of the cell with an aqueous solution must be quite large so that during the time of passage of the nanopore, at least several DNA molecules can pass through the pore. In this case, the time of passage of the molecule around the electrodes will increase, and the time it takes to read the molecule will be relatively long. The movement of the electrodes can only be adjusted mechanically. Regulation of the pore movement speed by electromagnetic forces is probably impossible, because firstly the electrodes themselves are charged and secondly, they must move in the field created by the potential $V_{0}$.

\section{DNA Molecules Reading (Translocation) Time}

Time for DNA molecules reading (translocation) can be set by the duration of 
the electric pulse on the metal electrodes (see [6]). Denote this time by $t_{r}$.

By the sizes of gap between nucleotides $d_{0}=0.34 \mathrm{~nm}$ (see [35]) we can determine the time of absence of the applied impulses on the metallic electrodes [6]. This time can be calculated according to formula $t_{g}=d_{0} / v$, or

$$
t_{g}=d_{0}\left[\frac{m_{0}+\rho C_{D} A l_{0}}{2\left(m_{0} g l_{0}-q V_{0}\right)}\right]^{1 / 2},
$$

The period of the pulses applied to the gold electrodes will be

$$
T=t_{r}+t_{g} .
$$

The frequency of the base's passes (i.e. electrical pulses applied to lateral gold electrodes) will be

$$
f=\frac{1}{2 \pi T}=\frac{1}{2 \pi\left(t_{r}+t_{g}\right)} .
$$

Here $t_{r}$ is the time for DNA molecules bases (A, T, C or G) reading (translocation), and

$$
t_{r}=h_{A(T, C, G)} / \mathcal{U},
$$

$h_{A(T, C, G)}$ is the vertical size of corresponding nucleotides $\left(h_{A(T, C, G) \text { max }}=h_{C}\right.$ $=0.583 \mathrm{~nm}$ [see Appendix]), $d_{0}=0.34 \mathrm{~nm}$ is the size of gap between nucleosides [35].

Example 1: $V_{0}=0.001 \mathrm{~V}$ and $l_{0}=0.02 \mathrm{~m}, N_{n}=40 ; 1-\mathcal{E}=10^{-6}$, and $v=633 \mathrm{~nm} / \mathrm{s}$, we get $f \approx 110 \mathrm{~Hz}, t_{r} \approx 9 \times 10^{-4} \mathrm{~s}=0.9 \mathrm{~ms} ; 1-\mathcal{E}=10^{-5}$, and $v=2000 \mathrm{~nm} / \mathrm{s}$, we get $f \approx 345 \mathrm{~Hz}, t_{r} \approx 3 \times 10^{-4} \mathrm{~s}=0.3 \mathrm{~ms}$.

Example 2: $V_{0}=0.001 \mathrm{~V}$ and $l_{0}=0.02 \mathrm{~m}, N_{n}=1000 ; 1-\mathcal{E}=10^{-6}$, and $v=3170 \mathrm{~nm} / \mathrm{s}$, we get $f \approx 547 \mathrm{~Hz} ; t_{r} \approx 2 \times 10^{-4} \mathrm{~s}=0.2 \mathrm{~ms} ; 1-\mathcal{E}=10^{-5}$, and $v=10,000 \mathrm{~nm} / \mathrm{s}$, we get $f \approx 1725 \mathrm{~Hz} ; t_{r} \approx 6 \times 10^{-5} \mathrm{~s}=0.06 \mathrm{~ms}$.

If $t$ is the passing full time for one DNA molecule through nanopore and $N_{n}$ is the number of bases groups in DNA, we can write

$$
v=\frac{l_{\mathrm{DNA}}}{t},
$$

where

$$
l_{\mathrm{DNA}}=N_{n}\left(h_{\mathrm{A}}+h_{T}+h_{G}+h_{C}\right)+\left(N_{n}-1\right) d_{0}
$$

is the DNA total length consisting only groups with four bases. Here $h_{A}=$ $0.480 \mathrm{~nm}, h_{T}=0.504 \mathrm{~nm}, h_{G}=0.514 \mathrm{~nm}$ and $h_{C}=0.583 \mathrm{~nm}$ are the sizes in direction of $Z$ axis of adenine, thymine, guanine and cytosine, correspondingly (see Appendix).

In the other hand

$$
t=N_{n} t_{r}+\left(N_{n}-1\right) t_{g}
$$

For example, for the case $t_{r}=3 \times 10^{-4} \mathrm{~s}, t_{g}=5.37 \times 10^{-4} \mathrm{~s}$ and $N_{n}=40$, we get $t \approx 0.031 \mathrm{~s}$.

It is quite large and measurable time for reading of DNA sequencing. 
We can control value of $t_{r}$ and $f$ by the choice magnitudes of $V_{0}$ and $l_{0}$. For comparison note that in Ref. [7] the reasonable value of the DNA translocation speed is near to $0.01-1 \mathrm{~ms}$ per base considered. It is more short time compared with $0.031 \mathrm{~s}$. Note that all of the biological and synthetic nanopores have barrels of $\sim 5 \mathrm{~nm}$ (which is considerably longer than the base-to-base distance of $3.4 \AA$ ) in thickness and accommodate $\sim 10$ - 15 nucleotides at a time. It is, therefore, impossible to achieve single-base resolution using blockage current measurements. In addition, the average rate at which a polymer typically translocates through a nanopore is on the order of 1 nucleotide/ $\mu$ s (i.e., on the order of $\mathrm{MHz}$ detection), which is too fast to resolve.

\section{Conclusions}

Solid-state nanopore DNA sequencing modified method is developed and presented. The nanogap is made on gold electrodes in the form of nanowires or nanoribbons. A detailed analysis of the dynamics of a DNA molecule in an aqueous solution is carried out. For the first time in analytical calculations, all forces acting on DNA molecules are taken into account. The movement of DNA in aqueous solution is regulated by the potential applied to reference electrode. Taking into consideration that DNA moves under gravity, electrophoretic and drag forces of the expression for the DNA translocation speed are calculated and analyzed. The conditions for decreasing the DNA translocation speed or increasing reading time are received. Carried numerical calculations show that increasing the reading time is associated with the problem of very subtle regulation of the electric field strength acting on DNA molecule.

Based on the modified method proposed above it is possible to successfully decrease molecule translocation time and increase its reading time. It will give possibility of enlarging the passing time of tunnel current through gold-nucleotide-gold junction and more precisely determine and identify nucleotide type crating an electrical bridge between electrodes [6]. Our results therefore potentially suggest a realistic, inherently design-specific, high-throughput nanopore DNA sequencing device/cell as a de-novo alternative to the existing methods.

The amount of tunnel current which can pass through the nanopore at any given moment therefore varies depending on whether the nanopore is bounded by an A, T, C or G nucleotide. The change in the current through the nanopore as the DNA molecule passes through the nanopore represents a direct reading of the DNA sequence. Such behavior is ordinary. Analyses of the tunnel currents responsible for nitrogenous bases in DNA are developed and presented by us in [6].

It has been shown that one can control value of the DNA molecules bases reading time and the frequency of the electrical pulses applied to lateral electrodes by the choice of magnitude of the potential of the reference electrode. It is shown that DNA reading time can be quite large and measurable value in order to $10^{3}$ bases/s. The conditions of lengthening of the reading time are determined. 


\section{Author Contribution Information}

All authors participated in the statement of the problem and discussion of the results. L. Gasparyan, I. Mazo and F. Gasparyan conducted literature review. F. Gasparyan and V. Simonyan made calculations and participated in the writing of the text of the article.

\section{Conflicts of Interest}

The authors declare no conflicts of interest regarding the publication of this paper.

\section{References}

[1] Munshi, A. (2012) DNA Sequencing-Methods and Applications. InTech, London.

[2] Rincon-Restrepo, M., Mikhailova, E., Bayley, H. and Maglia, G. (2011) Controlled Translocation of Individual DNA Molecules through Protein Nanopores with Engineered Molecular Brakes. Nano Letters, 11, 746-750. https://doi.org/10.1021/nl1038874

[3] Howorka, S., Cheley, S. and Bayley, H. (2001) Sequence-Specific Detection of Individual DNA Strands Using Engineered Nanopores. Nature Biotechnology, 19, 636-639. https://doi.org/10.1038/90236

[4] Zahid, O.K., Wang, F., Ruzicka, J.A., Taylor, E.W. and Hall, A.R. (2016) Sequence-Specific Recognition of microRNAs and Other Short Nucleic Acids with Solid-State Nanopores. Nano Letters, 16, 2033-2039.

https://doi.org/10.1021/acs.nanolett.6b00001

[5] Clamer, M., Höfler, L., Mikhailova, E., Viero, G. and Bayley, H. (2014) Detection of 3'-end RNA Uridylation with a Protein Nanopore. ACS Nano, 8, 1364-1374. https://doi.org/10.1021/nn4050479

[6] Gasparyan, L., Mazo, I., Simonyan, V. and Gasparyan, F. (2020) Study of Molecular Junctions Metal-DNA-Metal for DNA Sequencing. Journal of Contemporary Physics (Armenian Academy of Sciences), 55, 77-86.

https://doi.org/10.3103/S1068337220010119

[7] Fologea, D., Uplinger, J., Thomas, B., McNabb, D.S. and Li, J. (2005) Slowing DNA Translocation in a Solid-State Nanopore. Nano Letters, 5, 1734-1737.

https://doi.org/10.1021/nl051063o

[8] Peng, H.B. and Ling, X.S.S. (2009) Reverse DNA Translocation through a Solid-State Nanopore by Magnetic Tweezers. Nanotechnology, 20, Article ID: 185101. https://doi.org/10.1088/0957-4484/20/18/185101

[9] McCormick, B.W. (1979) Aerodynamics, Aeronautics, and Flight Mechanics. John Wiley \& Sons, Inc., New York. https://en.wikipedia.org/wiki/Drag_coefficient

[10] He, Y., Tsutsui, M., Fan, C., Taniguchi, M. and Kawai, T. (2011) Controlling DNA Translocation through Gate Modulation of Nanopore Wall Surface Charges. ACS Nano, 5, 5509-5518. https://doi.org/10.1021/nn201883b

[11] Meller, A. (2003) Dynamics of Polynucleotide Transport through Nanometre-Scale Pores. Journal of Physics: Condensed Matter, 15, R581-R607. https://doi.org/10.1088/0953-8984/15/17/202

[12] Meller, A., Nivon, L., Brandin, E., Golovchenko, J. and Branton, D. (2000) Rapid Nanopore Discrimination between Single Oligonucleotide Molecules. Proceedings 
of the National Academy of Sciences of the United States of America, 97, 1079-1084. https://doi.org/10.1073/pnas.97.3.1079

[13] Mathe, J., Aksimentiev, A., Nelson, D.R., Schulten, K. and Meller, A. (2005) Orientation Discrimination of Single-Stranded DNA inside the $\alpha$-Hemolysin Membrane Channel. Proceedings of the National Academy of Sciences of the United States of America, 102, 12377-12382. https://doi.org/10.1073/pnas.0502947102

[14] Chang, S., Huang, S., He, J., Liang, F., Zhang, P., Li, S., Chen, X., Sankey, O. and Lindsay, S. (2010) Electronic Signatures of All Four DNA Nucleosides in a Tunneling Gap. Nano Letters, 10, 1070-1075. https://doi.org/10.1021/nl1001185

[15] Nakane, J., Wiggin, M. and Marziali, A. (2004) A Nanosensor for Transmembrane Capture and Identification of Single Nucleic Acid Molecules. Biophysical Journal, 87, 615-621. https://doi.org/10.1529/biophysj.104.040212

[16] Tropini, C. and Marziali, A. (2007) Multi-Nanopore Force Spectroscopy for DNA Analysis. Biophysical Journal, 92, 1632-1637. https://doi.org/10.1529/biophysj.106.094060

[17] Wanunu, M., Chakrabarti, B., Mathe, J., Nelson, D.R. and Meller, A. (2008) Orientation-Dependent Interactions of DNA with an $\alpha$-Hemolysin Channel. Physical Review E, 77, Article ID: 031904. https://doi.org/10.1103/PhysRevE.77.031904

[18] Payne, C.M., Zhao, X., Vlcek, L. and Cummings, P. (2008) Molecular Dynamics Simulation of ss-DNA Translocation between Copper Nanoelectrodes Incorporating Electrode Charge Dynamics. The Journal of Physical Chemistry B, 112, 1712-1717. https://doi.org/10.1021/jp077483e

[19] Branton, D., Deamer, D.W., Marziali, A. and Bayley, H. (2008) The Potential and Challenges of Nanopore Sequencing. Nature Biotechnology, 26, 1146-1153. https://doi.org/10.1038/nbt.1495

[20] Gasparyan, L., Mazo, I., Simonyan, V. and Gasparyan, F. (2019) DNA Sequencing: Current State and Prospects of Development. Open Journal of Biophysics, 9, 169-197. https://doi.org/10.4236/ojbiphy.2019.93013

[21] Gasparyan, L., Mazo, I., Simonyan, V. and Gasparyan, F. (2019) ISFET Based DNA Sensor: Current-Voltage Characteristic and Sensitivity to DNA Molecules. Open Journal of Biophysics, 9, 239-253. https://doi.org/10.4236/ojbiphy.2019.94017

[22] Gasparyan, L., Mazo, I., Simonyan, V. and Gasparyan, F. (2020) Noises and Signal-to-Noise Ratio of Nanosize EIS and ISFET Biosensors. Open Journal of Biophysics, 10, 1-12. https://doi.org/10.4236/ojbiphy.2020.101001

[23] Clarke, J., Wu, H.-C., Jayasinghe, L., Patel, A., Reid, S. and Bayley, H. (2009) Continuous Base Identification for Single-Molecule Nanopore DNA Sequencing. Nature Nanotechnology, 4, 265-270. https://doi.org/10.1038/nnano.2009.12

[24] Butler, T.Z., Gundlach, J.H. and Troll, M. (2007) Ionic Current Blockades from DNA and RNA Molecules in the $\alpha$-Hemolysin Nanopore. Biophysical Journal, 93, 3229-3240. https://doi.org/10.1529/biophysj.107.107003

[25] Perrin, M.L., Verzijl, C.J., Martin, C.A., Shaikh, A.J., Eelkema, R., van Esch, J.H., van Ruitenbeek, J.M., Thijssen, J.M., van der Zant, H.S. and Dulic, D. (2013) Large Tunable Image-Charge Effects in Single-Molecule Junctions. Nature Nanotechnology, 8, 282-287. https://doi.org/10.1038/nnano.2013.26

[26] Xiang, D., Jeong, H., Lee, T. and Mayer, D. (2013) Mechanically Controllable Break Junctions for Molecular Electronics. Advanced Materials, 25, 4845-4867. https://doi.org/10.1002/adma.201301589

[27] Vladyka, A., Perrin, M.L., Overbeck, J., Ferradas, R.R., Garcia-Suarez, V., Gan- 
tenbein, M., Brunner, J., Mayor, M., Ferrer, J. and Calame, M. (2019) In-Situ Formation of One-Dimensional Coordination Polimers in Molecular Junction. Nature Communications, 10, 262. https://doi.org/10.1038/s41467-018-08025-9

[28] Binnig, G., Rohrer, H., Gerber, Ch. and Weibel, E. (1982) Surface Studies by Scanning Tunneling Microscopy. Physical Review Letters, 49, 57-61. https://doi.org/10.1103/PhysRevLett.49.57

[29] Binnig, G., Quate, C.F. and Gerber, C. (1986) Atomic Force Microscope. Physical Review Letters, 56, 930-933. https://doi.org/10.1103/PhysRevLett.56.930

[30] Chassaing, P., Antonia, R.A., Anselmet, F., Joly, L. and Sarkar, S. (2002) Variable Density Fluid Turbulence (Fluid Mechanics and Its Applications). Vol. 69, Springer, Berlin. https://doi.org/10.1007/978-94-017-0075-7

[31] Mandelkern, M., Elias, J.G., Eden, D. and Crothers, D.M. (1981) The Dimensions of DNA in Solution. Journal of Molecular Biology, 152, 153-161. https://doi.org/10.1016/0022-2836(81)90099-1

[32] Meller, A., Nivon, L. and Branton, D. (2001) Voltage-Driven DNA Translocations through a Nanopore. Physical Review Letters, 86, 3435-3438. https://doi.org/10.1103/PhysRevLett.86.3435

[33] Smolyanitsky, A., Yakobson, B.I., Wassenaar, T.A., Paulechka, E. and Kroenlein, K. (2016) $\mathrm{A} \mathrm{MoS}_{2}$-Based Capacitive Displacement Sensor for DNA Sequencing. ACS Nano, 10, 9009-9016. https://doi.org/10.1021/acsnano.6b05274

[34] Steinbock, L.J. and Radenovic, A. (2015) The Emergence of Nanopores in Next-Generation Sequencing. Nanotechnology, 26, Article ID: 074003. https://doi.org/10.1088/0957-4484/26/7/074003

[35] Watson, J.D. and Crick, F.H. (1953) Molecular Structure of Nucleic Acids; a Structure for Deoxyribose Nucleic Acid. Nature, 171, 737-738. https://doi.org/10.1038/171737a0

[36] Pyykkö, P. and Atsumi, M. (2009) Molecular Double-Bond Covalent Radii for Elements Li-E112. Chemistry: A European Journal, 15, 12770-12779. https://doi.org/10.1002/chem.200901472 


\section{Appendix}

For calculations of nucleosides sizes, we use data for the covalent radii some elements presented in Table A1 [36].

Table A1. The covalent radii some elements in $\mathrm{nm}$ [36].

\begin{tabular}{cccc}
\hline Element & Single bond & Double bond & Triple bond \\
\hline $\mathrm{H}$ & 0.032 & - & - \\
$\mathrm{C}$ & 0.075 & 0.067 & 0.060 \\
$\mathrm{~N}$ & 0.071 & 0.060 & 0.054 \\
$\mathrm{O}$ & 0.063 & 0.052 & 0.053 \\
\hline
\end{tabular}

Let's denote the vertical size of the nucleotides through $h$, and the horizontal size-through 1 . Indexes $\mathrm{H}-\mathrm{C}$ (or $\mathrm{C}-\mathrm{H}$ ), $\mathrm{C}=\mathrm{N}$ etc. means single and double bonds between corresponding elements.

Adenine $\left(\mathrm{C}_{5} \mathrm{H}_{5} \mathrm{~N}_{5}\right)$

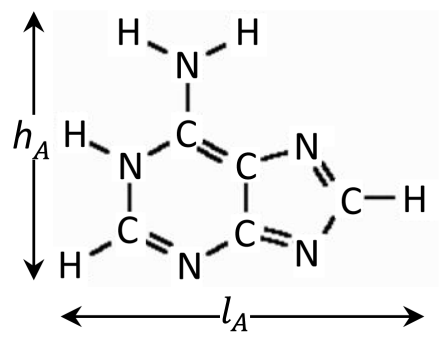

$$
\begin{aligned}
l_{A}= & l_{\mathrm{H}-\mathrm{C}} \cos 30+l_{\mathrm{C}=\mathrm{N}} \cos 30+l_{\mathrm{N}-\mathrm{C}} \cos 30+l_{\mathrm{C}=\mathrm{N}} \cos 18+l_{\mathrm{N}-\mathrm{C}} \sin 36+l_{\mathrm{C}-\mathrm{H}} \\
= & {[0.107+0.127+0.146] \times 0.866+0.127 \times 0.9511 } \\
& +0.146 \times 0.5878+0.107 \\
= & 0.3291+0.1208+0.094+0.107=0.651 \mathrm{~nm}
\end{aligned}
$$

$$
\begin{aligned}
h_{\mathrm{A}}= & h_{\mathrm{C}=\mathrm{N}} \sin 30+h_{\mathrm{C}-\mathrm{N}}+h_{\mathrm{N}-\mathrm{C}} \sin 30+h_{\mathrm{C}-\mathrm{N}}+h_{\mathrm{N}-\mathrm{H}} \sin 30 \\
= & (0.067+0.06+0.075+0.071+0.032+0.071) \times 0.5 \\
& +2 \times(0.071+0.075) \\
= & 0.188+0.292=0.48 \mathrm{~nm}
\end{aligned}
$$

\section{Guanine $\left(\mathrm{CH}_{5} \mathrm{~N}_{5} \mathrm{O}\right)$}

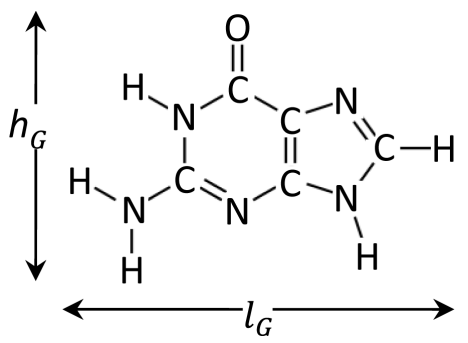




$$
\begin{aligned}
l_{G}= & h_{\mathrm{H}-\mathrm{N}} \cos 30+h_{\mathrm{N}-\mathrm{C}} \cos 30+h_{\mathrm{C}=\mathrm{N}} \cos 30+h_{\mathrm{N}-\mathrm{C}} \cos 30 \\
& +l_{\mathrm{C}-\mathrm{N}} \cos 18+l_{\mathrm{N}-\mathrm{C}} \sin 36+l_{\mathrm{C}-\mathrm{H}} \\
= & (0.032+0.071+0.071+0.075+0.067+0.06+0.071+0.075) \times 0.866 \\
& +(0.075+0.071)(0.9511+0.5878)+(0.075+0.032) \\
= & 0.4521+0.2247+0.082=0.784 \mathrm{~nm} \\
h_{G}= & h_{\mathrm{H}-\mathrm{N}}+h_{\mathrm{N}-\mathrm{C}} \cos 60+h_{\mathrm{C}-\mathrm{N}}+h_{\mathrm{N}-\mathrm{C}} \cos 60+h_{\mathrm{C}=\mathrm{O}} \\
= & 0.032+0.071+2 \times 0.5 \times(0.075+0.071) \\
& +0.075+0.071+0.067+0.052 \\
= & 0.514 \mathrm{~nm}
\end{aligned}
$$

Thymine $\left(\mathrm{C}_{5} \mathrm{H}_{6} \mathrm{~N}_{2} \mathrm{O}_{2}\right)$

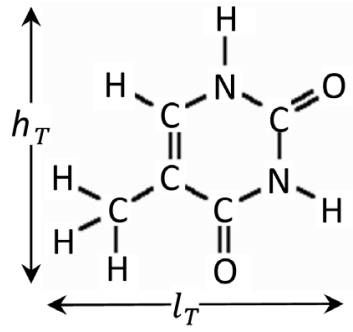

$$
\begin{aligned}
l_{T}= & \left(l_{\mathrm{H}-\mathrm{C}}+l_{\mathrm{C}-\mathrm{C}}+l_{\mathrm{C}-\mathrm{C}}+l_{\mathrm{C}-\mathrm{N}}+l_{\mathrm{N}-\mathrm{H}}\right) \times \cos 30 \\
= & (0.032+0.075+0.075+0.075+0.075+0.075 \\
& +0.075+0.071+0.071+0.032) \times 0.866 \\
= & 0.656 \mathrm{~nm} \\
h_{T}= & h_{\mathrm{O}=\mathrm{C}}+h_{\mathrm{C}-\mathrm{C}} \sin 30+h_{\mathrm{C}=\mathrm{C}}+h_{\mathrm{C}-\mathrm{N}} \sin 30+h_{\mathrm{N}-\mathrm{H}} \\
= & 0.052+0.067+(0.075+0.075+0.075+0.071) \times 0.5 \\
& +0.067+0.067+0.071+0.032 \\
= & 0.504 \mathrm{~nm}
\end{aligned}
$$

\section{Cytosine $\left(\mathrm{C}_{4} \mathrm{H}_{5} \mathrm{~N}_{3} \mathrm{O}\right)$}

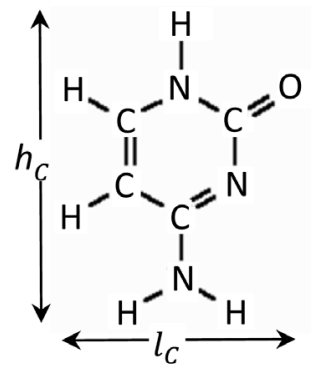

$$
\begin{aligned}
l_{C}= & l_{\mathrm{H}-\mathrm{C}} \cos 30+l_{\mathrm{C}-\mathrm{N}} \cos 30+l_{\mathrm{N}-\mathrm{C}} \cos 30+l_{\mathrm{C}=\mathrm{O}} \cos 30 \\
= & (0.032+0.075+0.075+0.071+0.071 \\
& +0.075+0.067+0.052) \times 0.866 \\
= & 0.449 \mathrm{~nm}
\end{aligned}
$$




$$
\begin{aligned}
h_{C}= & h_{\mathrm{H}-\mathrm{N}} \sin 30+h_{\mathrm{N}-\mathrm{C}}+h_{\mathrm{C}-\mathrm{C}} \sin 30+h_{\mathrm{C}=\mathrm{C}}+h_{\mathrm{C}-\mathrm{N}} \sin 30+h_{\mathrm{N}-\mathrm{H}} \\
= & (0.032+0.071+0.075+0.075+0.075+0.071) \times 0.5 \\
& +0.071+0.075+0.067+0.067+0.071+0.032 \\
= & 0.583 \mathrm{~nm}
\end{aligned}
$$

